María Cristina Ascuntar Rivera*

\title{
Evolución contextual del dibujo en diseño industrial ${ }^{1}$
}

\section{Cómo citar:}

Ascuntar Rivera, M.C. (2018). Evolución conceptual del dibujo en diseño industrial. Designia, 5(2), 121-127.

*Diseñadora Industrial de la Universidad de Nariño; Especialista en Gerencia de Diseño de la Universidad Jorge Tadeo Lozano/Universidad de Nariño; Magíster en Diseño y Creación Interactiva de la Universidad de Caldas. Docente investigadora de la Universidad de Nariño (Pasto). E-mail: cristinascuntar@udenar.edu.co ORCID: 0000-0003-2671-3909
Palabras clave:

Analógico, contexto, dibujo, diseño industrial, digital, evolución.

\section{Key words:}

Analog, context, drawing, industrial design, digital, evolution.

Recibido: $12 / 06 / 2018$

Aceptado: 17/10/2018

\section{Resumen:}

En la sección “Disertaciones sobre Diseño" del Vol 5, Núm. 2 de la revista Designia, la autora invitada presenta un artículo corto de reflexión, en el que se discute acerca de la enseñanza y aprendizaje del dibujo en la disciplina del diseño industrial y la coyuntura que supone el abordaje del mismo desde el contexto digital y análogo.

En la disertación, se desarrolla una mirada acerca de la evolución del dibujo como eje de la formación del diseñador industrial y los nuevos entornos pedagógicos que supone la enseñanza de esta asignatura en la actualidad. Si bien los contextos educativos actuales exigen el uso instrumentos tecnológicos, no cambia la función primordial del dibujo, como herramienta de representación y exteriorización de las ideas. 


\begin{abstract}
In the section "Dissertations on Design" of Vol 5, Issue 2 of the Designia magazine, the guest author presents a short article of reflection, which discusses the teaching and learning of drawing in the discipline of industrial design and the conjuncture which implies the approach of the same from the digital and analogous context.

In the dissertation, the topic is developed from the evolution of drawing as the axis of the training of the industrial designer and the new pedagogical environments that the teaching of this subject implies today. Although current educational contexts require the use of technological tools, the primordial function of drawing does not change as a tool for representation and externalization of ideas.
\end{abstract}

\section{Introducción}

A partir de la configuración del diseño industrial como disciplina en la segunda década del siglo XX, el dibujo se ha constituido en una herramienta crucial para el diseñador, debido a que es el medio por excelencia que facilita la exteriorización de las ideas; por tal razón, se ha establecido como una estructura de comunicación ineludible en los procesos proyectuales asociados al diseño industrial. Este hecho, permite destacar la habilidad de dibujar como una competencia fundamental en la formación del estudiante de diseño industrial, puesto que potencia la capacidad para expresar sus ideas, que más tarde se materializarán en objetos de uso cotidiano. Sin embargo, a mediados de la década de los 60 `s del siglo pasado, ocurrió un hecho sin precedentes en torno al dibujo en diseño: se trata de la aparición del primer paquete de diseño asistido por computador
(CAD), suceso que provocó discrepancias entre las formas tradicionales de representación y las nuevas alternativas digitales, estableciéndose como punto de partida para la evolución contextual del dibujo.

El presente documento, es una breve reflexión sobre el estado del dibujo dentro del entorno pedagógico que involucra la formación del diseñador industrial, con el fin de propiciar la disertación acerca de nuevos paradigmas en la enseñanza del dibujo en diseño y facilitar la estructuración de panoramas emergentes en relación con las coyunturas entre el dibujo analógico y digital. Una de las conclusiones de esta indagación, se refiere a la visualización de nuevos entornos pedagógicos del dibujo, donde se logre articular las técnicas tradicionales con las digitales, favoreciendo procesos de enseñanza y aprendizaje que incluyan el uso de herramientas tecnológicas, sin desatender las funciones primordiales del dibujo en diseño. 
Desde tiempos prehistóricos, el dibujo se ha constituido en un medio adecuado para representar los idearios, pensamientos, emociones, modos de vida y en general la cotidianidad del ser humano; prueba de ello, son las representaciones de dibujos desde las primigenias etapas en las que se inició el desarrollo humano, atravesando por distintas fases del progreso de la sociedad. Con el transcurrir del tiempo, el dibujo se ha constituido en una estructura comunicativa utilizada por distintos artistas, arquitectos y diseñadores, ya que es el medio más oportuno para la representación de las ideas que surgen en el pensamiento y tienden a desaparecer rápidamente, debido a la limitada capacidad de la memoria activa del ser humano (Tversky, 2002).

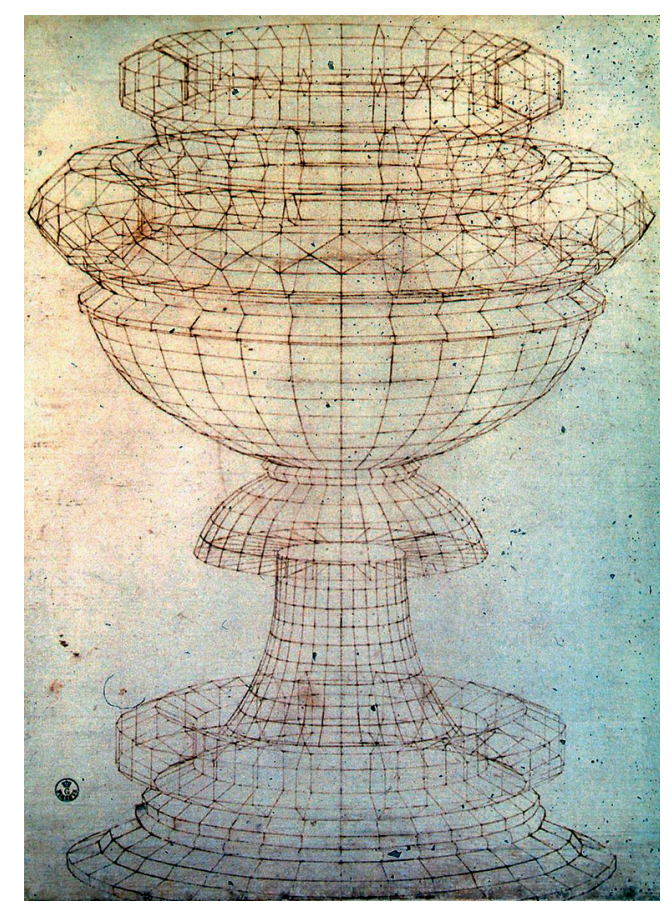

Figura 1. Dibujo de cáliz en perspectiva realizado por Paolo Uccello en 1430. Fuente: Art Special Day, http://www.artspecialday. com/9art/2018/06/14/paolo-uccello-pittore-fiaba/
De acuerdo con los planteamientos del inglés Alan Pipes, especializado en aplicaciones de gráficos por ordenador para diseño de productos, el diseñador mantiene una relación especial con los dibujos, ya que es el medio más inmediato para transmitir información acerca de complejas formas tridimensionales, dotando de personalidad y facilidad de uso a nuevos productos, quizá desconocidos para el consumidor (Pipes, 1989). A partir de la anterior inferencia, se deduce que la relación entre el diseñador industrial y el dibujo se ha tornado cada vez más estrecha, por cuanto incide notablemente en la actividad proyectual, puesto que, en las fases iniciales del proceso, el diseñador hace uso del dibujo para comunicar las ideas que más tarde se concretarán en objetos de diseño. Sin embargo, han sido perceptibles las mutaciones contextuales del dibujo en el diseño industrial, dado que, en la actualidad, existe una amplia gama de posibilidades que ofrece el creciente avance tecnológico respecto al dibujo, donde diariamente se percibe el incremento en el desarrollo de diversos soportes para la representación, junto a los considerables progresos del software para tales fines.

La evolución contextual del dibujo fue más palpable cuando ocurrió la mutación del tradicional soporte de papel a la pantalla. La línea de tiempo revela que la aparición del primer paquete de dibujo asistido por computador (CAD) fue en el año de 1965, convirtiéndose en un hecho trascendental para la evolución del dibujo en diseño; el Sketchpad, fue desarrollado por Iván Sutherland como parte de su tesis doctoral en el MIT y contenía muchas de las características esperadas en los sistemas modernos de dibujo bidimensional, además, permitía la manipulación directa de objetos 
gráficos y evidenciaba que era un proceso interactivo, puesto que el diseñador podía comunicarse instantáneamente con el ordenador utilizando un lápiz óptico y un tubo de rayos catódicos (Manovich, 2013). Según Helen Armstrong (2016) cuando Sutherland creó el Sketchpad "abrió las puertas del castillo de la computación a los ingenieros y diseñadores" (pág. 37).

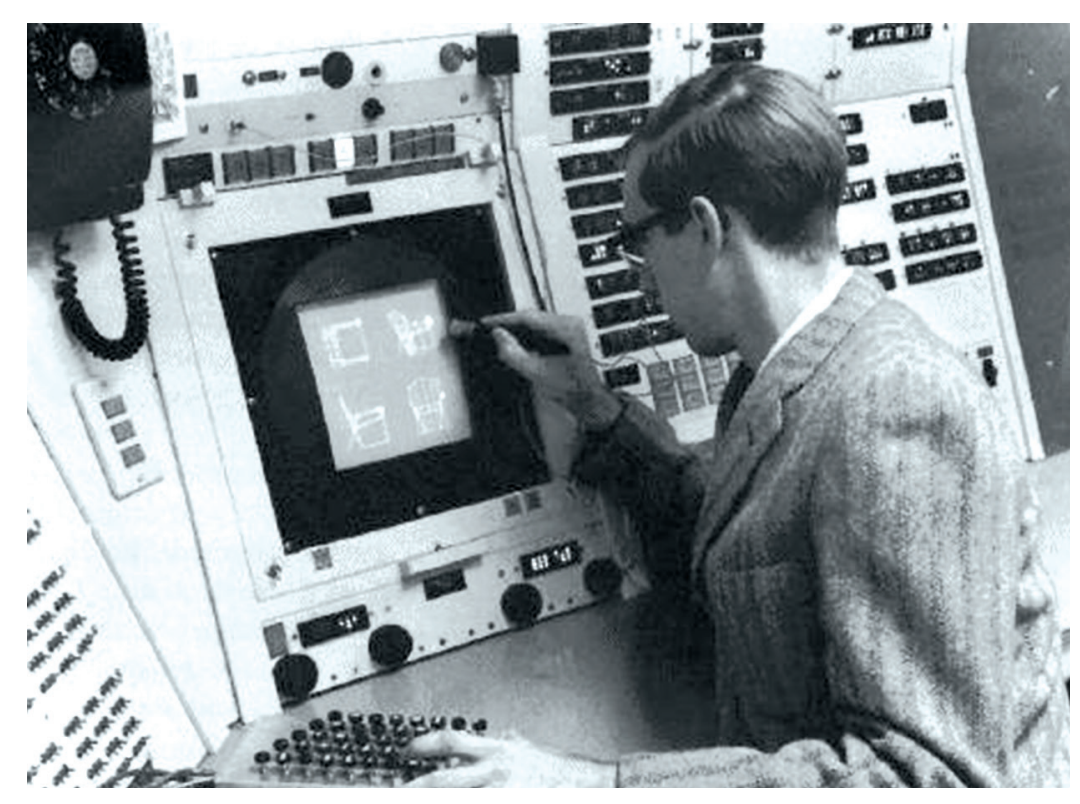

Figura 2. Ivan Sutherland utilizando el Sketchpad (1965).

Fuente: La computación gráfica,

El surgimiento del CAD, ha otorgado grandes beneficios principalmente a los diseñadores, dadas las características de su trabajo, sin embargo, no puede evadirse el hecho de que se suscitó una confrontación entre el dibujo analógico y digital, y en la misma medida, provocó la mutación de los contextos del dibujo en diseño industrial, incidiendo directamente en los entornos pedagógicos relacionados a las prácticas del dibujo. Ya desde la década de los 80's Alan Pipes (1989) reflexionaba acerca del futuro del dibujo en diseño, manifestando que, si el ordenador se usa correctamente, en alguna medida contribuirá a devolver a los diseñadores un grado de control del que no gozan desde que la industrialización comenzó a fragmentar el ciclo diseño-producción. Sin embargo, tal prescripción, llegaría al punto en donde la existencia del dibujo a mano y su presencia en los procesos de diseño, se vería subestimada, frente a las magníficas posibilidades que ofrece el dibujo por computador. Desde otra instancia, Marc 
Treib (2008) pone al descubierto que la productividad digital exige un precio demasiado alto al diseño, cuando desplaza a los medios de representación tradicionales y para contrarrestar esta situación, es conveniente que se produzca un consenso, donde haya una profunda complementariedad en el uso de los medios analógicos y digitales, contrarrestando las limitaciones de cada uno.

Siendo evidentes las divergencias entre el dibujo analógico y digital, se hace perentoria la construcción de nuevos espacios pedagógicos para la enseñanza del dibujo en el diseño industrial. Para tal fin, es prioritario tener en cuenta que las generaciones actuales y futuras de estudiantes de diseño, se enfrentan a nuevas plataformas de interacción relacionadas a la práctica del dibujo. Según Alejandro Folga (2016), “de la mano de los constantes avances tecnológicos, los nativos digitales abandonan el lápiz y el papel para abrazar las herramientas informáticas"' (pág. 2). Las primeras manifestaciones de esta situación ocurren en los ámbitos académicos y más tarde, se trasladan a la práctica profesional. Al respecto, Kevin Henry (2012) manifiesta la importancia del dibujo analógico para la construcción de un buen dibujo digital, ya que el ordenador necesita entradas específicas y en ese sentido no visualiza milagrosamente lo que se está pensando, mientras que el tradicional dibujo a mano permite representar las ideas antes que desaparezcan del pensamiento. En consecuencia, se hace más palpable la necesidad de construir nuevos espacios de enseñanza que permitan articular las prácticas de representación tradicionales y digitales, donde se integre el uso de herramientas tecnológicas que coadyuven a potenciar los procesos de enseñanza y aprendizaje del dibujo en el diseño industrial. Investigaciones relacionadas al tema, como la propuesta metodológica FER (familiarización exposición - reafirmación) de Juan Antonio Islas, brinda una perspectiva de transformación de los contextos pedagógicos del dibujo en el diseño industrial, donde se manifiesta la relación cambiante entre el cerebro, la mano y ahora el ordenador (Islas Muñoz, 2016).

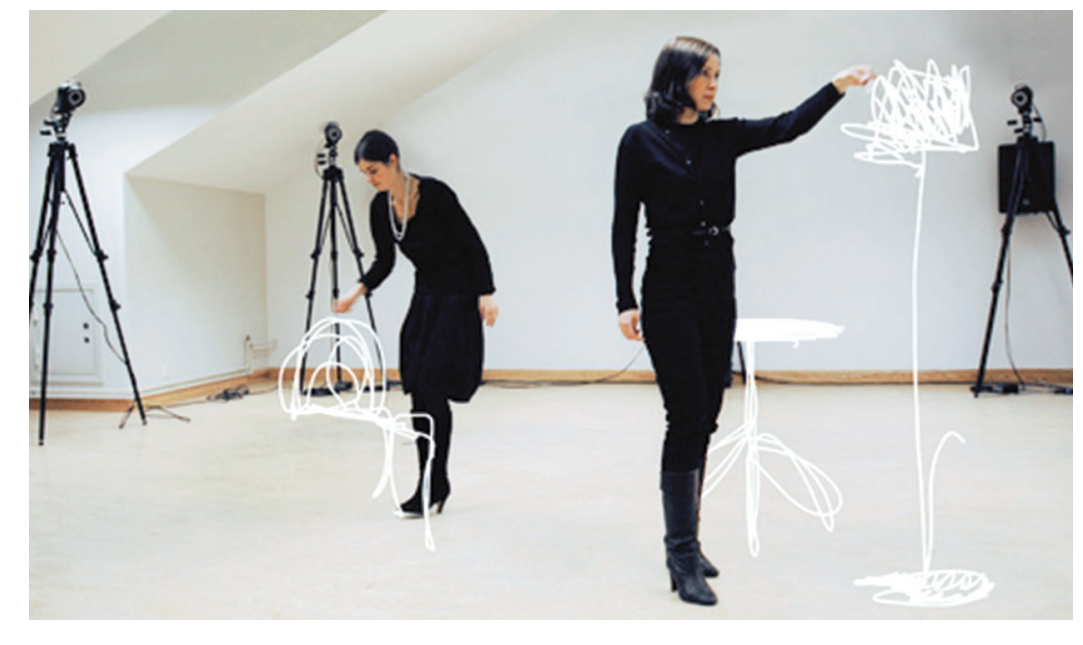

Figura 3. Sketch Furniture: realización de dibujos en el aire a mano alzada en el estudio de diseño sueco Front (2009). Fuente: Revista Código, http://www.revistacodigo.com 
Finalmente, es sustancial que la configuración de los nuevos contextos pedagógicos del dibujo se realice con la participación de los estudiantes y docentes, donde las herramientas tecnológicas se conviertan en mecanismos que permitan potenciar las capacidades de estudiante para comunicar a través del dibujo. Al realizar una mirada de la tecnología hacia la didáctica, la construcción de una nueva metodología debe abordarse a partir de los medios de representación tradicionales, pero sin desatender las propuestas innovadoras desde el desarrollo de las TIC, que propician la construcción de ambientes pedagógicos (Quintana Guerrero, Barbosa, \& Cuenca, 2011). Si bien, los nuevos contextos brindan multiplicidad de posibilidades para el dibujo en diseño industrial, es conveniente el establecimiento de un balance entre lo analógico y digital, ya que el dibujo analógico, es una capacidad innata del ser humano, en tanto que el dibujo digital es el resultado del constante avance tecnológico y del permanente desarrollo social. En último término, los contextos pedagógicos del dibujo en diseño, se constituyen en los espacios idóneos para la investigación respecto al tema, donde se visualiza un vasto campo de acción de estudiantes, docentes y en general de toda la comunidad académica del diseño, donde los procesos de enseñanza y aprendizaje del dibujo, sean acordes con las demandas de las nuevas sociedades y con la evolución que cada día afronta el diseño como disciplina. 


\section{REFERENCIAS BIBLIOGRÁFICAS}

Armstrong, H. (2016). Digital Design Theory: readings from the field. New York: Princeton. Architectural Press.

Folga, A. (2016). Dibujo a mano alzada y medios gráficos digitales. Congreso Latinoamericano de Enseñanza del Diseño. Buenos Aires: Universidad de Palermo.

Henry, K. (2012). Dibujo para diseñadores de producto: de la idea al papel. Barcelona: Promopress.

Islas Muñoz, J. A. (2016). Aplicaciones tecnológicas para la enseñanza del dibujo para diseño industrial. Actas de Diseño. Buenos Aires: Universidad de Palermo.

Manovich, L. (2013). El software toma el mando. Barcelona: Editorial UOC.

Pipes, A. (1989). Diseño tridimensional: del boceto a la pantalla. Barcelona: Parramón Ediciones.

Quintana, B., Barbosa, I., \& Cuenca, A. (2011). Dibujo tridimensional y diseño: una mirada desde la academia. Bogotá: Editorial Fundación Universidad Autónoma de Colombia.

Treib, M. (2008). Drawing/thinking: confronting an electronic age. Londres: Routledge.

Tversky, B. (2002). What do sketches say about thinking? Stanford University.

Van den Toorn, M., \& Have, R. (2012). The role of hand drawing in basic desig. International Conference on Engineering and Mathematics, ENMA (págs. 72-88). Delft: Delft University of Technology. 